\title{
The 5th International Conference on Tumor Microenvironment: Progression, Therapy and Prevention Versailles, France, October 20-24, 2009
}

\section{Conference Summary}

\author{
Suresh Mohla • Isaac P. Witz
}

Published online: 18 February 2010

(C) Springer Science+Business Media B.V. 2010

The 5th International Conference on Tumor Microenvironment: Progression, Therapy, and Prevention was held at the Palais des Congres de Versailles, Versailles, France from October 20 to October 24, 2009. The beautiful conference venue was highly suitable for personal interactions between participants, a most important factor in a scientific gathering.

With about 700 scientists representing 43 countries, this conference was the largest Tumor Microenvironment (TME) conference ever held. One Hundred and Eighty oral presentations were delivered in 6 plenary and 14 symposium sessions. In addition, 221 posters were presented.

The Versailles conference was a truly multidisciplinary event where the focal issue was approached and discussed thoroughly by specialists from a wide spectrum of biomedical sciences. The conference met the intention of the program committee to solidify the realization about the significance of the tumor microenvironment in tumor progression as well as to create a friendly forum that promotes a critical review of novel basic findings, and of innovative clinical studies pertaining to the cancer microenvironment.

Of significance was the fact that a large fraction (36\%) of the participants was graduate students and post-doctoral fellows making their first steps in TME research. This demonstrated that young scientists consider the area of

\section{S. Mohla}

Division of Cancer Biology, National Cancer Institute,

National Institutes of Health,

Bethesda, MD, USA

e-mail:mohlas@mail.nih.gov

\section{P. Witz $(\square)$}

Department of Cell Research \& Immunology,

Faculty of Life Sciences, Tel Aviv University,

Ramat Aviv,

Tel Aviv 69978, Israel

e-mail: IsaacW@tauex.tau.ac.il
TME as being of prime importance in the cancer process and worthwhile of focusing on. In order to familiarize the younger generation with the roots of the TME research area, one of us (IPW) summarized the major developmental stages of this research area (Witz IP., Cancer Microenviron. 2 Suppl 1:9-17, 2009).

In a plenary poster session, five scientists whose posters were selected by a review committee as best posters presented their results. These scientists were awarded with "best poster prizes" (Fig. 1). Two "best poster prizes" were sponsored by the European Association for Cancer Research and the other 3 were sponsored by the American Association of Cancer Research, the National Cancer Institute of France and the International Cancer microenvironment Society. Two citations for excellent posters were also awarded.

The major goal of all cancer researchers world wide, including, of course the tumor microenvironmentalists is to cure cancer or at least block its progression. Indeed results of the last decade clearly demonstrate that drugs that modify or interfere with malignancy-promoting interactions between tumor cells and their microenvironment such as anti angiogenesis drugs, signaling inhibitors or anti inflammatory drugs exert beneficial influences on cancer patients.

The majority of topics discussed in the Versailles conference dealt with basic and translational aspects of tumor-microenvironment interactions.

What are the prerequisites of successful therapeutic/ prevention targeting strategies? In attempts to answer this question we should remember that the tumor is a complex entity as borne out by the following facts:

1. There is a multitude of candidate microenvironmental factors

2. The signaling cascades in the TME are complex 


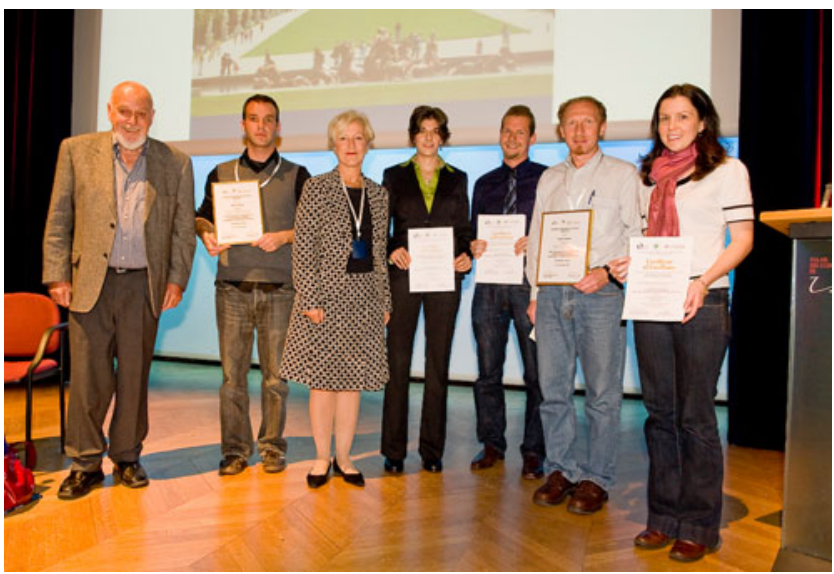

Fig. 1 Awarding of "Best Poster" Prizes at the Poster Session. From left to right: Isaac P. Witz, Conference Chairperson; Dominique Arsenault; "Best Poster" Selection Committee Chairperson, Catherine Sautes-Fridman; Jennifer Isaacs, Niels Halama; Andrei Bakin; Emily Paterson

3. There is a cross-talk among cascades

4. Tumors are heterogeneous.

These realities bring home the point that specific molecule(s) or signal transduction pathway(s) are but components of an immense network and therefore will not be effective targets.

To make cancer therapies more effective we need to explore and overcome the hurdles presented by the above 4 points. This requires the generation of novel insights to TME interactions requiring both reductionist but mainly system biology approaches and the use of emerging technologies such as laser capture, imaging, OMICS, nanotechnology as well as novel resources and models.

Cancer is an ecosystem composed of tumor cells and of non tumor cells residing in the microenvironment of the tumor cells or recruited to this site. Secreted molecules originating either in the tumor cells themselves or in the non tumor cells present in the TME are important constituents of this microenvironment.

Numerous lectures and posters presented at the conference put a great emphasis on the characterization and functions of the non tumor cell component of the TME in the cancer initiation-progression-metastasis continuum. Endothelial cells, bone marrow derived cells (BMDC), cancer associated fibroblasts (CAF), adipocytes and lymphatic and myeloid immunocytes as well as a variety of growth factors, cytokines, chemokines, proteases, other critical enzymes, as well as extracellular matrix proteins and their bioactive fragments were thoroughly discussed. Several presentations dealt with tumor progenitor or cancer stem cells and the relevance of premetastatic niches.

Essentially all major cancer sites - solid tumors as well as hematopoietic malignancies were well represented at this meeting including multiple myeloma, leukemias, breast, prostate, renal, colorectal, lung, pancreatic, glioblastoma, endometrial, ovarian and cervical cancer.

Below is a summary of some interesting topics discussed in the conference.

Intra-tumor Heterogeneity. Several talks dealt with the characterization of necrotic avascular, perinecrotic areas and the leading edge of the tumors. The overall perception is that tumors demonstrate considerable intra-tumor heterogeneity. For example the leading edge has more tumor cells that seemingly undergo epithelial to mesenchymal transition (EMT) and has more adipocytes than fibroblasts while fibroblasts predominate in the center of the tumor.

Key Players in EMT and Its Reversion. Several talks on EMT indicated that a variety of growth factors or chemokines are able to stimulate EMT. Equally noteworthy was the demonstration that neutralization of Her2 in estrogen receptor negative human breast cancer cell line can reverse the process so that mesenchymal cells transform and acquire epithelial cell morphology.

Cell Migration and Tumor Associated Macrophages (TAM). Several talks were focused on tumor-cell migration during invasion and progression. There were also other talks where investigators showed that during single cell migration of tumor cells, macrophages are generally (over $50 \%$ of the time) found adjacent to migrating cells, and that TAM were found around the blood vessels. It was also shown that there was a correlation between circulating tumor cell number and the number of TAM around the tumor.

Molecular Signatures of Non tumor Cells in the TME. Several talks focused on the unique gene signatures of tumor or cancer associated fibroblasts, bone marrow derived cells and tumor associated macrophages. There is ongoing research on other microenvironmental non-tumor cells such as $\mathrm{B}$ and $\mathrm{T}$ cells. A signature of such cells is also being developed. This aspect of research is still evolving and it should not be too long before signatures of non tumor cells in the TME are used translationally in the clinical setting.

Tumor cells and non tumor cells in the TME can have overlapping signaling cascades. Several soluble factors secreted by tumor cells and by non tumor cells in the TME may initiate signaling cascades both in the tumor cells as well as in a number of non tumor cells including BMDC, CAF, lymphocytes, TAM and adipocytes. For example estrogen mediated signaling in endothelial progenitor cells or toll like receptor- 4 may transduce signals in CAF. A number of non tumor cell types in the TME appear to modulate inflammation, matrix remodeling, angiogenesis, invasion, migration or metastasis. It appears that tumor cells 
and/or non tumor cells in the TME are able to generate an overall invasive and/or a cell survival phenotype. The questions that emerge from these observations are: do tumors have a built in redundancy? Will a systems biology approach allow us to identify whether there are some unifying mechanisms?

Bone Marrow Derived Cells (BMDC). (a) the recruitment of BMDC by tumors was found to be at a low level but a chronic phenomenon. It was also reported that BMDC are recruited early in the process of tumorigenesis before the onset of angiogenesis. In contrast, following therapy, e.g. after the administration of anti angiogenic therapy, there is an acute but robust recruitment of BMDC into the tumor. (b) BMDC may play an important role in establishing the premetastatic niche. (c) BMDCs increase MMP production in the TME and increase angiogenesis; (d) BMDC can block anti-tumor responses by $\mathrm{T}$ cells.

Other topics that that were dealt with include the following:

Hypoxia. The progression of solid tumors is universally characterized by a state of hypoxia, low extracellular $\mathrm{pH}$, and high glycolysis. These factors being functionally connected via hypoxia have an enormous potential to promote tumor progression. Indeed numerous studies indicate that hypoxia plays a critical and fundamental role in metastasis formation. Hypoxia drives tumor progression by influencing both the tumor cells as well as the TME. One of the major effects of hypoxia on the microenvironment is the stimulation of angiogenesis. Hypoxia alters the expression of hypoxia inducible factor (HIF), a heterodimeric transcription factor, composed of constitutively expressed HIF- $1 \beta$ and HIF- $1 \alpha$ or HIF- $2 \alpha$ subunits. Control of HIF-1 function occurs primarily through oxygendependent enzymatic hydroxylation of the $\alpha$ subunit. Under hypoxia, the $\alpha$ subunit is stabilized, translocates to the nucleus, and dimerizes with HIF-1 $\beta$. The stable dimer stimulates the transcription of a large variety of key target genes controlling cell survival, anaerobic metabolism, migration, and angiogenesis, to name just a few. These genes would confer upon tumor cells various metastasispromoting characteristics. Hypoxia, its related signaling and associated phenotypic changes were well represented at this conference.

Chemoine-chemokine Receptor Axes. Several reports addressed the role of chemokines in cancer progression. Emerging results seem to indicate that certain chemokines or their receptors are tumor specific. For example CCR7 is most often associated with brain tumors and leukemia while CCR 3, 4 and 10 are associated with skin cancer and lymphomas.
Escape from Anti Tumor Immunity and Inflammation. Numerous reports show that tumor cells may escape immune surveillance and many tumor types demonstrate an inflammatory microenvironment. Several participants suggested that the inflammatory microenvironment and immune escape should be added to the list of Cancer Hallmarks.

Bone Metastasis. It became obvious in the meeting that an immense progress was made in studying interactions between bone metastasizing tumor cells and the bone microenvironment. The vicious cycle wherein tumor cell and the bone microenvironment demonstrate reciprocal interactions provides not only an excellent paradigm to delineate novel mechanisms, but also an ideal experimental setting to target multiple cell types such as osteoblasts, osteoclasts, bone endothelial cells, mesenchymal and hematopoietic stem cells as well as tumor cells. Progress in this research area is likely to enable the targeting of multiple cell types in other cancers as well.

Targeting Non-tumor Cells in the TME. There are a number of trials targeting non tumor cells in the TME including endothelial cells (avastin), breast cancer fibroblasts (aromatase inhibitors) and memory $\mathrm{T}$ cells. This is a highly promising indicator that targeting of the tumor microenvironment may provide vital additional targets in combating cancer.

\section{Goals and Priorities of TME Research}

One of the high priorities of TME research is to generate a functional atlas of the tumor microenvironment in different organs and determine key changes that occur to explain organ specific metastasis. Such an effort will (a) identify unique features of non-tumor cells in the TME of different organs, (b) identify whether there are common mechanisms that occur independent of tumor types, (c) identify tumorspecific features of each cancer type, which in turn, will shed light on organ-specific metastasis or tissue tropism; (d) resolve issues related to stem cell/progenitor cells: definition of these cell types, whether stem cells are required for tumorigenesis.

Another area of priority is, as mentioned above, to develop strategies to target the tumor microenvironment we are getting closer to achieving this in the bone microenvironment and perhaps soon in other primary and metastatic cancer sites as well.

\section{Unresolved Issues}

As we gain novel and deeper insights into the tumormicroenvironment interactions that either retard tumor 
progression or, more often, promote it, new questions emerge. Moreover unresolved issues that need a solution remain in this ever expanding area of the tumor microenvironment. Several presentations left us with open questions and unresolved issues. Below is a summary of these questions and issues.

Cancer Associated Fibroblasts. What is the origin of CAF (tumor? bone marrow?); CAF are heterogeneous. We need markers to differentiate CAF subtypes; we need to validate the role of CAF in tumor formation, migration, invasion, angiogenesis, and metastasis.

Bone Marrow Derived Cells. BMDC are heterogeneous. We need markers to differentiate BMDC subtypes and clinical validation data on the role of BMDC in tumor progression.

Tumor Associated Macrophages. What is the origin and the role of TAM in tumor onset and metastasis?

Tumor Dormancy. There are currently at least two approaches to study dormancy (i) Isolation and characterization of disseminated dormant tumor cells and delineating mechanism of their activation, and (ii) using experimental models or isolated human cancer cells and delineating mechanism that induce dormancy. Both approaches are useful and should continue.

Metabolism. Cancer metabolism is an emerging and important aspect of tumor microenvironment. Molecules such as HIF 1- $\alpha$, carbonic anhydrase or uncoupling proteins are a subject of intense research which is ready to be exploited.

Cell Fusion. Recent reports suggest that cell fusion may confer drug resistance in multiple myeloma and that chronic inflammation can promote fusion of BMDC to intestinal progenitor cells. More data and mechanisms of these phenomena are needed.

Embryonic Transcription Factors. Several examples of embryonic transcription factors (e.g. Hoc 10a and Hox 5) being lost in tumor cells were presented. There are other reports in the literature that show that several other transcription factors such as snail, slug and twist, are over expressed in tumors. An embryonic stem cell factor such as Nodal is aberrantly expressed in aggressive breast cancer and melanoma cells. Isolated examples were presented to indicate that non-tumor cells in the TME may express an embryonic phenotype. For example it was noted that the gene signature of TAM closely resembles the gene signature of embryonic macrophages. Whether other non tumor cells in the tumor microenvironment show similar tendency to express an embryonic phenotype is an area for future investigation.

Cellular Architecture. Several presentations dealt with the architecture of the tumor microenvironment and matrix remodeling. The role of cellular architectural changes in the tumor microenvironment during cancer initiationprogression-metastasis continuum should be investigated further. Another question related to the architecture topic is the question if there are microenvironments within a tumor microenvironment? This challenging and interesting area should be subject for further investigation.

Micro RNA Biology. The information on micro RNA is still primordial and at the discovery stage. However in several investigators are planning to target these noncoding RNAs.

Imaging of the Tumor Microenvironment. Imaging modalities are used to analyze changes in the tumor microenvironment with respect to: premalignancy or early cancer stages, the fate of tumor cells during intravasation and extravasation, identification of micrometastases or resistant tumors and follow therapeutic responses. These promising areas are of great importance and should be advanced.

Novel Diagnostic and Prognostic Biomarkers of Non Tumor Cells in the TME. Several presentations dealt with molecular signatures of various non-tumor cells such as fibroblasts and TAM. Such signatures seem to correlate with tumor progression. This promising research area should be expanded.

Targeting Non Tumor Cells in the TME. Tumor microenvironment targeting can be used as prevention strategy i.e. to block progression of pre neoplastic lesions; or create cytostatic effects to tame an aggressive cancer into a benign disease; or induce tumor dormancy; or cause the regression of established tumors; or reverse therapeutic resistance. Several pre-clinical reports on targeting of non tumor cells in the TME showed that such an approach is feasible and that it may be beneficial to tumor bearers in the clinical setting as a neoadjuvant.

Stem Cell Niche. In the healthy individual, stem cells reside in special niches. The cells in the niche deliver signals that maintain the stem cells and control their special properties. Several presentations dealt with stem cell niches and the establishment of a premetastatic niche. It was demonstrated that a certain population of BMDC is recruited by factors 
derived from the primary tumor to specific secondary sites. The presence of the BMDCs in these sites changes the local microenvironment and creates the premetastatic niche in which early metastasis develops.

Further studies on the early cellular and molecular events in cancer dissemination and organ selectivity are needed. The elucidation of the mechanisms leading to the establishment of the pre-metastatic niche and the identification of the molecules involved in this process may lead to new approaches to detect and prevent metastasis.

Acknowledgments We wish to express our appreciation to the 3 organizing partners of the Versailles conference, the American Association for Cancer Research (AACR), the International Cancer Microenvironment Society and the National Cancer Institute of France (INCa) for organizing an outstanding conference. 